\title{
MIMO Wireless Communications
}

EZIO BIGLIERI

Universitat Pompeu Fabra

ROBERT CALDERBANK

Princeton University

ANTHONY CONSTANTINIDES

Imperial College of Science, Technology and Medicine

ANDREA GOLDSMITH

Stanford University

AROGYASWAMI PAULRAJ

Stanford University

H. VINCENT POOR

Princeton University 


\section{Contents}

List of contributors

page ix

Preface

Acknowledgements

$\mathrm{xi}$

Notation

xiii

xiv

1 Introduction

1.1 MIMO wireless communication

1.2 MIMO channel and signal model

1.3 A fundamental trade-off

1.4 MIMO transceiver design

1.5 MIMO in wireless networks

1.6 MIMO in wireless standards

1.7 Organization of the book and future challenges

1.8 Bibliographical notes

References

2 Capacity limits of MIMO systems 24

$\begin{array}{lll}2.1 & \text { Introduction } & 24\end{array}$

2.2 Mutual information and Shannon capacity 25

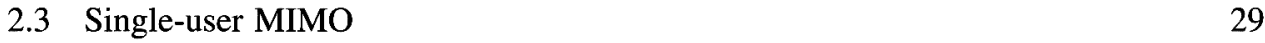

2.4 Multi-user MIMO $\quad 49$

2.5 Multi-cell MIMO $\quad 66$

2.6 MIMO for ad hoc networks $\quad 69$

$\begin{array}{lll}2.7 & \text { Summary } & 75\end{array}$

$\begin{array}{lll}2.8 & \text { Bibliographical notes } & 77\end{array}$

$\begin{array}{ll}\text { References } & 77\end{array}$

3 Precoding design $\quad 88$

3.1 Transmit channel side information $\quad 89$

3.2 Information-theoretic foundation for exploiting CSIT 95

$\begin{array}{ll}3.3 \text { A transmitter structure } & 100\end{array}$

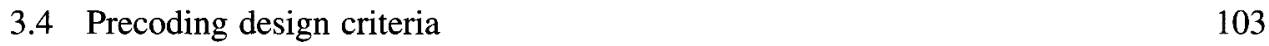

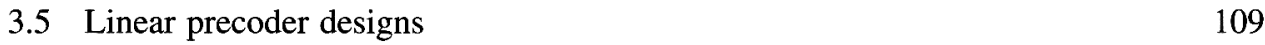

3.6 Precoder performance results and discussion 122

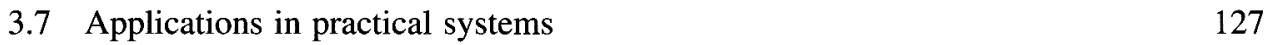

$\begin{array}{ll}3.8 \text { Conclusion } & 132\end{array}$

3.9 Bibliographical notes 133 
Appendix 3.1

133

References

135

4 Space-time coding for wireless communications: principles and applications

4.1 Introduction

4.2 Background

4.3 Space-time coding principles

4.4 Applications

161

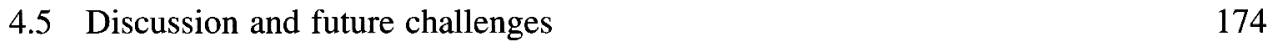

$\begin{array}{lll}4.6 & \text { Bibliographical notes } & 176\end{array}$

$\begin{array}{ll}\text { Appendix 4.1 Algebraic structure: quadratic forms } & 177\end{array}$

$\begin{array}{ll}\text { References } & 180\end{array}$

5 Fundamentals of receiver design 186

$\begin{array}{ll}5.1 \text { Introduction } & 186\end{array}$

5.2 Reception of uncoded signals $\quad 186$

$\begin{array}{lll}5.3 & \text { Factor graphs and iterative processing } & 190\end{array}$

5.4 MIMO receivers for uncoded signals 202

5.5 MIMO receivers for coded signals 206

$\begin{array}{lll}5.6 & \text { Some iterative receivers } & 223\end{array}$

$\begin{array}{ll}5.7 \text { Bibliographical notes } & 226\end{array}$

$\begin{array}{ll}\text { References } & 227\end{array}$

6 Multi-user receiver design 230

$\begin{array}{lll}6.1 \text { Introduction } & 230\end{array}$

6.2 Multiple-access MIMO systems 231

6.3 Iterative space-time multi-user detection 245

6.4 Multi-user detection in space-time coded systems 256

6.5 Adaptive linear space-time multi-user detection 271

$\begin{array}{lll}6.6 & \text { Summary } & 288\end{array}$

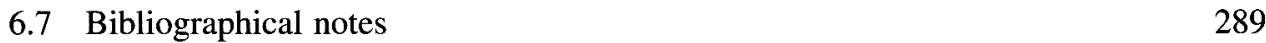

$\begin{array}{ll}\text { References } & 289\end{array}$

Bibliography $\quad 293$

$\begin{array}{ll}\text { Index } & 315\end{array}$ 